\title{
Light-Based Circadian Rhythm Control: Entrainment and Optimization
}

\author{
Jiaxiang Zhang ${ }^{\mathrm{a}}$, Wei Qiao ${ }^{\mathrm{b}}$, John T. Wen ${ }^{\mathrm{b}}$, Agung Julius ${ }^{\mathrm{b}}$

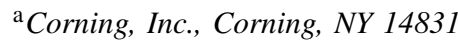 \\ ${ }^{\mathrm{b}}$ Rensselaer Polytechnic Institute, Troy, NY 12180
}

\begin{abstract}
Light is a strong synchronizer for circadian rhythm - the 24-hour biological oscillation in plants, insects, and mammals. This paper considers the circadian entrainment problem for a popular circadian oscillation model (the Kronauer model) by using light intensity as the control input. This problem is commonly encountered by shift workers and international travellers - how to shift the phase of one's circadian rhythm by a specified amount, preferably as fast as possible? We consider three approaches: 1. Periodic entrainment: use the light/dark cycle corresponding to the desired circadian rhythm as the light input. 2. Optimal entrainment: use light input to shift the circadian rhythm to the desired state in minimum time. 3. Feedback entrainment: use circadian state feedback to adjust light input. For feedback entrainment, we consider two cases: active lighting control which can inject artificial lighting on demand and subtractive lighting control which only blocks the ambient lighting. For the periodic entrainment, which is used a baseline for comparison, we apply the harmonic balance method to assess the existence of a stable periodic solution, and verify the result by simulation. For the minimum time entrainment, we present an efficient solution to the two-point boundary value problem and show that active lighting control significantly reduces the entrainment time from the baseline. The feedback algorithm augments the periodic entrainment with a circadian state feedback to account for modeling error and noise. Results from this study provide new insight and guideline to light intensity control for circadian rhythm regulation.
\end{abstract}

Key words: circadian rhythm, circadian entrainment, lighting control, optimal control, phase control.

\section{Introduction}

Terrestrial species have adapted to the 24-hour daily light/dark cycle, called the circadian rhythm. For humans, circadian rhythms are regulated by the suprachiasmatic nuclei (SCN) in the hypothalamus in the brain. The SCN governs a wide range of biological cycles, from cell division, hormone production, to behavior (e.g., sleep-wake) [1,2]. Light is a strong synchronizer of the circadian rhythm. It affects SCN through the intrinsically photosensitive Retinal Ganglion Cells (ipRGC) in the retina. These cells are distinct from photoreceptors responsible for vision (rods and cones) and are most sensitive to the blue portion of spectrum (centers around 460nm) versus the photopic spectrum which is centered around $550 \mathrm{~nm}$. Circadian rhythms manifest in humans through the sleep/wake cycle, hormone

\footnotetext{
Corresponding author John T. Wen

Email addresses: zhangjiaxiang035@gmail.com (Jiaxiang Zhang), michael.f.jovi@gmail.com (Wei Qiao), wenjerpi.edu (John T. Wen), agung@ecse.rpi.edu (Agung Julius).
}

production and levels of daytime/nighttime performance and alertness. Lack of synchrony between the master clock in SCN and the external environment, referred to as circadian misalignment, could lead to serious health issues ranging from increased sleepiness and decreased attention span, gastrointestinal disorders, to increased risk for cancer, diabetes, obesity, and cardiovascular disorders [3]. The circadian disruption may be caused by, for example, irregular sleep patterns of soldiers in the battlefield, artificial deprivation of light of submariners or mine workers, frequently shifted sleep-wake cycles of night nurses, and shifted lightdark cycles for travelers across multiple time zones.

Numerous mathematical models have been proposed to describe the interaction between light intensity and the circadian oscillation $[4,5]$. Empirical nonlinear oscillator model has been long proposed [6], with subsequent modification by Richard Kronauer et al. [7,8]. The Kronauer model describes the relationship between light intensity and the oscillation of human core body temperature, which is an acceptable phase marker of the circadian system. The model is a modified Van der Pol oscillator with a period of 24.2 hours. Alternative mechanistic biomolecular models based on protein interac- 
tions have been developed for various organisms, including the 3-state Neurospora model [9], 10-state Drosophila model [10-12], 19-state Drosophila model [11,13] and 74state mammalian model [14]. As demonstrated in [15], the empirical model may be considered as the asymptotic case of the biomolecular model, at least for Drosophila, in an averaged sense. Reduced order modeling approaches for biomolecular model have also been proposed in [16].

Artificial light has long been proposed as a means to entrain the circadian rhythm. A commonly used tool is the phase response curve (PRC) which plots the steady state phase shift as a function of the time of the day at which a light pulse with a given amplitude and duration is applied. The PRC may be generated experimentally using test subjects, or numerically with a chosen simulation model. An open loop circadian entrainment method has been proposed based on the PRC constructed from the Kronauer model to design a light-dark pattern for jet lag treatment [17]. A 10state circadian oscillation model of Drosophila was used to construct the PRC and closed loop model predictive control based on the phase measurement [18]. The PRC approach has also been extended to the synchronization of an oscillator networks under a global driving cue such as sunlight [19]. General circadian rhythm control methods based on PRC have been proposed in [20,21]. However, PRC only considers dynamics on the periodic orbit which depends on the external input such as light pulse amplitude and duration [22]. Relaxation dynamics off the periodic solution is considered in [23] with the light input pulses optimized to achieve fast convergence. If the initial circadian state starts on the open loop entrained periodic orbit, the effect of amplitude deviation from the orbit is small. In this case, we expect the PRC-based method to produce similar results as the full phase plane method in this paper. This is indeed the case and has been demonstrated in [21]. If there is a large deviation from the periodic orbit, due to the initial condition or high light input amplitude, then the full phase dynamics need to be taken into account as in this paper.

This paper focuses on light-based control for the Kronauer model which includes the off-orbit behavior not contained in the PRC models but avoids the high order dynamics in the biomolecular models. The Kronauer model has been used to design light-based control using model based methods such as model predictive control [24] and optimal control [25]. We formulate the entrainment problem as a trajectory tracking problem with the reference trajectory given by the desired circadian oscillation. We consider three entrainment strategies:

(1) Periodic entrainment: The ambient periodic light/dark cycle of the reference oscillation is used as the entrainment input.

(2) Optimal entrainment: The light input is solved from an optimal control problem to align the circadian oscillation with the reference trajectory in minimum time.

(3) Feedback entrainment: The periodic entrainment is modified based on the circadian tracking error - through blockage when ambient light is present or light injection when ambient light is absent. We also consider the case when only blockage is allowed - we call this the subtractive entrainment.

The periodic entrainment is the commonly used default strategy in our daily living. For example, a traveler through multiple time zones would rely on the local light/dark cycle to combat jet lag. We will use it as a baseline comparison with other entrainment methods. We apply the harmonic balance method to analyze the existence and stability of the periodic solution. Harmonic balance [26, 27] has long been used in the analysis of nonlinear oscillators. Though it is an approximate method, it tends to work well when the linear portion of the system attenuates the effect of higher order harmonics. This is indeed the case for the Kronauer model under the free running (no light input) condition [28]. The harmonic balance method has also been applied in the systems biology context, e.g., chemical oscillations in gene regulatory networks [29]. In this paper, we extend harmonic analysis to light inputs under different conditions, including input duty cycles (e.g., people living in higher latitude locations), entrainment periods (submariners), and light intensity (cloudy versus sunny days). The analytic prediction, though approximate, matches well with the simulation result.

The optimal entrainment case is formulated as a time optimal control problem which involves the solution of a twopoint boundary value problem (TPBVP). We present an efficient solution strategy, involving a line search instead of the classical shooting or collocation methods. It also avoids searching for switching times as in [25]. The minimum time control significantly reduces the baseline periodic entrainment time. For example, for the most challenging case of 17 hours delay (or 7 hours advance), the baseline entrainment of 10 days is reduced to 4 days under optimal control. This work follows closely our paper in [30]. The feedback approach adjusts the light input based on the circadian tracking error. It modifies the baseline periodic input by adding artificial lighting when ambient lighting is absent or removes light input by blocking the ambient lighting. By using the stability analysis from the harmonic balance approach, we show that this additional lighting feedback does not affect the stability, but could speed up convergence. We show that the entrainment time for moderate phase delay or advance is close to the optimal cost. As a special case, we also consider the blockage-only strategy. This removes the need of active light control. Such controllable blockage of light in the circadian action spectrum (blue light), may be achieved by using, e.g., custom shaded glasses. We show that the entrainment time is near optimal for moderate phase advance (e.g., traveling from west to east). Implementation of circadian feedback requires the circadian state. State estimation using the Kronauer model and biometric sensors together with a particle filter has been proposed in [31]. We have applied linear parameter varying approach for state estimation in [32]. Phase estimation in the PRC framework is used in [20] for feedback light-based phase control. 
This paper presents new tools to analyze the Kronauer model for circadian rhythm, including new stability analysis (using harmonic balance) and new control methods (optimal control and feedback control). The results provide guidance to efficient entrainment for circadian phase shift.

\section{Problem Formulation}

\subsection{Model}

We consider the Kronauer model [33] for the circadian rhythm dynamics. It consists of a photoreceptor model, called the $L$-process, cascaded with a second order nonlinear oscillator, called the $P$-process. The $L$-process converts the light stimulus to a drive variable $u$, analogous to photopigments in retinal photoreceptor stimulated by incoming photons. There is a bleaching, or saturation, effect, which prevents further photon response until the photopigments are regenerated. In the Kronauer model, a simple first order population model is used to convert the light intensity $I$ to the circadian drive $u$ :

$$
\begin{aligned}
\alpha & =\alpha_{0}\left(\frac{I}{I_{0}}\right)^{p} \\
\dot{n} & =60[\alpha(1-n)-\beta n] \\
u & =G \alpha(1-n)
\end{aligned}
$$

where $\alpha_{0}=0.16 \mathrm{~min}^{-1}, \beta=0.013 \mathrm{~min}^{-1}, G=19.9, p=0.6$, $I_{0}=95001$ ux. For a fixed intensity light input $I, n$ converges to $\alpha /(\alpha+\beta)$ with rate $60(\alpha+\beta)$. As the dynamics of the $L$-process is much faster than that of the $P$-process (convergence is less than 1 minute), we focus on the dynamics of the $P$-process. As $n$ converges to a steady state, the corresponding circadian drive $u$ converges to $G \alpha \beta /(\alpha+\beta)$, which is a monotonically increasing function in $\alpha$. We limit the maximum light intensity at $I_{\max }=95001$ ux with the corresponding maximum drive $u_{\max }=0.2392$. Light intensity cannot be negative, so $u \geq 0$.

The circadian dynamics, the $P$-process, relates the drive input $u$ to the normalized core body temperature variation which is a good phase marker of the circadian system. The model for the $P$-process is given by (time unit is hour):

$$
\begin{aligned}
\dot{x} & =f_{0}(x)+f_{1}(x) u, \quad x=\left[\begin{array}{ll}
x_{1} & x_{2}
\end{array}\right]^{T} \\
f_{0} & =A x+\mu B g\left(B^{T} x\right) \\
A & =\frac{\pi}{12}\left[\begin{array}{cr}
\mu / 3 & 1 \\
-\left(24 /\left(.99729 \tau_{x}\right)\right)^{2} & 0
\end{array}\right], \quad B=\left[\begin{array}{l}
1 \\
0
\end{array}\right] \\
g\left(x_{1}\right) & =\frac{\pi}{12}\left(\frac{4}{3} x_{1}^{3}-\frac{256}{105} x_{1}^{7}\right) \\
f_{1}(x) & =\frac{\pi}{12}\left[\begin{array}{ll}
1 & q x_{2}+k x_{1}
\end{array}\right]^{T}\left(1-0.4 x_{1}\right)\left(1-0.4 x_{2}\right)
\end{aligned}
$$

where $\mu=0.13, k=0.55, q=0.33, \tau_{x}=24.2$ are constants empirically chosen so the model output $x_{1}$ matches physio- logical data (normalized core body temperature). The limit cycle in the phase plane, $\left(x_{1}, x_{2}\right)$, is approximately a unit circle (the describing function method predicts the limit cycle to be exactly the unit circle with period $24.13 \mathrm{hr}$ [34]). The oscillator rotates in the clockwise direction, with the $45^{\circ}$ direction $\left(x_{1}=x_{2}\right)$ corresponding to the mid-day $(12 \mathrm{pm})$. The model is depicted in block diagram form in Figure 1. Figure $! 2$ shows the input vector field, $f_{1}$, superimposed on the limit cycle. Since the light input is non-negative, the effect of light is mostly to add delay to the oscillation, except for the upper quarter of the limit cycle.

Note that the feedback loop consists of a single odd nonlinearity $g$, and the response of $B^{T}(j \omega I-A)^{-1} B$ has a sharp bandpass characteristics centered at $\omega=0.26 \mathrm{rad} / \mathrm{sec}$, or $T=$ $24.1 \mathrm{hr}$. These two characteristics suggest the harmonic balance method $[26,27]$ as a good tool to analyze the existence and stability of the limit cycle. The single nonlinearity is easily replaced by its describing function approximation, and the sharp bandpass characteristics implies that the harmonic balance is a reasonable approximation.

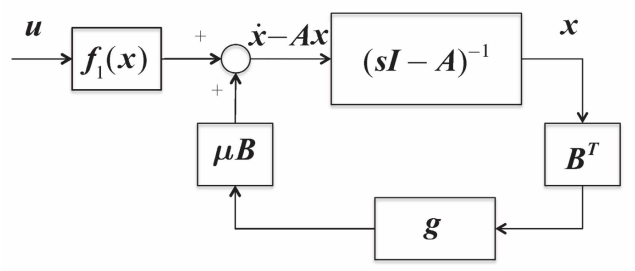

Fig. 1. The Kronauer model shown in the block diagram form.

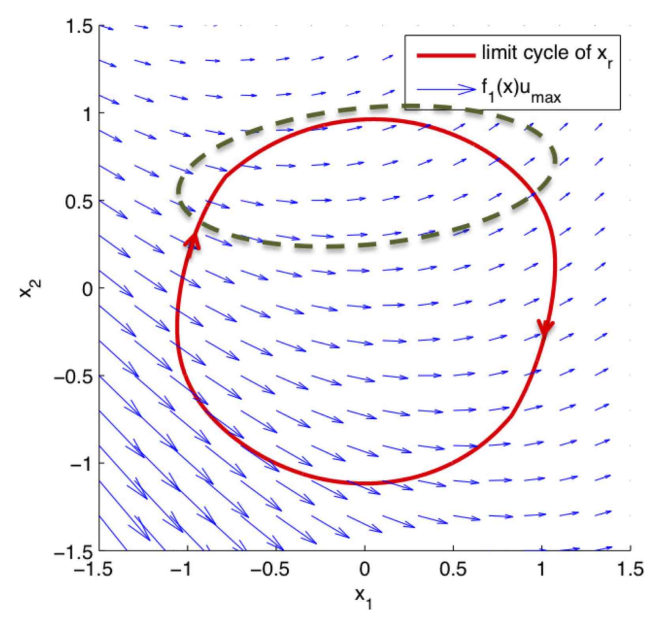

Fig. 2. The input drive function, $f_{1}(x)$, shown as a vector field, superimposed on the limit cycle. The circled portion at the top of the limit cycle indicates where light input will speed up the oscillation (shorten the period). For the rest of the limit cycle, light input will slow down the oscillation (lengthen the period) 


\subsection{Problem Statement}

The aim of this paper is to compare different light-based entrainment strategies for the Kronauer model. A motivating example is the travel through multiple time zones. The goal is to use light input to shift the traveler's circadian rhythm to that of the local population. Denote the state of the traveler's circadian rhythm as $x$ modeled by (2). Consider the local circadian rhythm given by $x_{r}$ governed by (2) with input $u_{r}$ :

$$
\dot{x}_{r}=f_{0}\left(x_{r}\right)+f_{1}\left(x_{r}\right) u_{r}(t)
$$

where $u_{r}$ is the local light cycle given by

$$
u_{r}(t)= \begin{cases}u_{\max } & \bmod (t, T) \in\left[t_{a}, t_{b}\right] \\ 0 & \bmod (t, T) \in[0, T] \backslash\left[t_{a}, t_{b}\right]\end{cases}
$$

with $t$ referring to the traveler's time at the starting location where $t_{a}, t_{b}$ are the starting time of the light on and light off, respectively in each period. If the starting location lighting is a $12 \mathrm{hr}-12 \mathrm{hr}$ pattern with light onset at $t_{0}$ (e.g., 6am), then the phase shift is $\left(t_{a}-t_{0}\right) 2 \pi / T$ (positive for delay, negative for advance). The duty cycle (percentage of the time of one period in which the light on signal is active.) of such on/off lighting is given by $d=\left(t_{b}-t_{a}\right) / T \cdot 100 \%$. If $t_{b}>T$, then the range where the light input is $u_{\max }$ consists of two pieces, $\left[0, t_{b}-T\right]$ and $\left[t_{a}, T\right]$. The goal is to find the light pattern $u(t), 0 \leq u(t) \leq u_{\max }$ to synchronize the traveler's circadian rhythm with the local circadian rhythm, i.e., choose $u(t)$ to drive $x(t) \rightarrow x_{r}(t)$.

\section{Entrainment Strategies}

\subsection{Periodic Entrainment}

The most common entrainment strategy is to use the periodic light/dark cycle, $u_{r}$, as the input. In the case of traveling through multiple time zones, the traveler just uses the natural daylight pattern of the local time zone for entrainment. In this case, the dynamics of the circadian rhythm is given by:

$$
\dot{x}=f_{0}(x)+f_{1}(x) u_{r}
$$

where $u_{r}$ is a period- $T$ input. Our goal is to ascertain the existence of a $T$-periodic solution, and if it exists, its local stability property. The exact analysis is difficult even for these simple nonlinear systems. The harmonic balance method is a popular approximation approach to analyze the existence and stability of periodic solutions in nonlinear systems [26]. For free-running system $(u=0)$, we have already shown in [28] that the so-called single-input describing function correctly predicts the existence of a periodic orbit close to a unit circle. With a periodic light input, we may extend the harmonic analysis to check for the existence of periodic solutions as well as their stability. The idea is to approximate all signals in the system by their constant and first order harmonics, i.e.,

$$
\begin{aligned}
\hat{x}_{1} & =a_{1} \sin \omega t+b_{1} \cos \omega t+c_{1} \\
\hat{x}_{2} & =a_{2} \sin \omega t+b_{2} \cos \omega t+c_{2} \\
\hat{g} & =\hat{g}_{0}+\hat{g}_{s} \sin \omega t+\hat{g}_{c} \cos \omega t \\
\hat{v} & =\hat{v}_{0}+\hat{v}_{s} \sin \omega t+\hat{v}_{c} \cos \omega t
\end{aligned}
$$

where $\hat{g}$ approximates $g\left(\hat{x}_{1}\right), \hat{v}$ approximates $v=f_{1}(\hat{x}) u$, and $\omega=2 \pi / T$ is given. Substituting these approximations into the system dynamics (2) and equating the coefficients of the harmonic components results in six equations to solve for the six unknown coefficients in $\left(\hat{x}_{1}, \hat{x}_{2}\right)$. Using this approach, we predict the existence of a periodic solution, and their approximate shape, for a large range of parameters: the duty cycle, $d$, entrainment period, $T$, and maximum light intensity, $u_{\max }$ of $u_{r}$. To determine the local stability of a predicted solution, we linearize the dynamics about the approximate solution:

$$
\delta \dot{x}=\underbrace{\left(A+B g^{\prime}\left(B^{T} \hat{x}(t)\right) B^{T}+\frac{\partial f_{1}(\hat{x}(t))}{\partial x} u(t)\right)}_{\triangleq \hat{A}(t)} \delta x
$$

where $\hat{A}(t)$ is $T$-periodic. The fundamental matrix over one period,

$$
\Phi(T) \triangleq e^{\int_{0}^{T} \hat{A}(t) d t},
$$

may be numerically evaluated and its eigenvalues determine the stability of the periodic orbit, and, if the orbit is stable, the local convergence rate. The detail approach is described in Appendix A. We shall see in Section 3.4 that the prediction result matches well with simulation.

\subsection{Optimal Entrainment}

Optimal entrainment aims to establish a lower bound on how fast light control can shift the circadian cycle by a specified phase. We pose this as an optimal control problem. Given the circadian dynamics (2) and the reference circadian trajectory (3) with specified initial state, find $u(t), 0 \leq u \leq u_{\max }$ to minimize the cost function

$$
J=t_{f}=\int_{0}^{t_{f}} 1 d t
$$

where $t_{f}$ is the entrainment time. The terminal state constraint is both state and time dependent:

$$
\psi\left(x\left(t_{f}\right), t_{f}\right)=x\left(t_{f}\right)-x_{r}\left(t_{f}\right)=0 .
$$

The necessary condition for this minimum time control problem may be readily stated using the Pontryagin Minimum Principle [35]. The Hamiltonian of the system is

$$
H=1+\lambda^{T}\left(f_{0}(x)+f_{1}(x) u\right)
$$


where $\lambda$ is the co-state and satisfies

$$
\dot{\lambda}=-\frac{\partial H}{\partial x}=\underbrace{-\frac{\partial\left(f_{0}(x)+f_{1}(x) u\right)^{T}}{\partial x}}_{\triangleq f_{2}(x, u)} \lambda .
$$

The optimal control $u^{*}$ minimizes $H$ and is given by the following "bang-off" control:

$$
u^{*}(x, \lambda)=\frac{u_{\max }}{2}\left(1-\operatorname{sgn}\left[\lambda^{T} f_{1}(x)\right]\right)
$$

Since $t_{f}$ is free, the terminal constraint (10) leads to the transversality condition:

$$
H\left(t_{f}\right)+\lambda^{T}\left(t_{f}\right) \frac{\partial \psi}{\partial t_{f}}=1+\lambda^{T}\left(t_{f}\right)\left(\dot{x}\left(t_{f}\right)-\dot{x}_{r}\left(t_{f}\right)\right)=0
$$

Substituting the optimal control into the state and co-state equations, we obtain the following two-point boundary value problem (TPBVP):

$$
\begin{aligned}
& \dot{x}=f_{0}(x)+f_{1}(x) u^{*}(x, \lambda) \\
& \dot{\lambda}=f_{2}\left(x, u^{*}(x, \lambda)\right) \lambda
\end{aligned}
$$

where $x(0)=x_{0}$ and $x\left(t_{f}\right)=x_{r}\left(t_{f}\right)$ are specified, and $t_{f}$ satisfies the transversality condition (14).

There are multiple techniques to solve the TPBVP numerically. One may regard the problem as having three unknowns, $\left(\lambda(0), t_{f}\right)$, and three algebraic equations, the terminal state constraint and transversality condition (14). Standard nonlinear minimization falls into local minima easily unless a good initial guess is available. Standard boundary value problem solver runs into numerical difficulty due to the unstable co-state equation propagation. Another approach is to convert the free terminal time problem to the fixed terminal time through the normalization $\tau=t / t_{f}$. This results in a $5^{\text {th }}$ order TPBVP. Direct numerical solution techniques such as the multiple shooting method [36] also requires a reasonably good initial guess of the state trajectory, which is difficult to obtain in general. Other approaches such as direct update of the switch times [37] and continuation method (starting from the solution for a linear oscillator and then propagating along the parameter to add in the nonlinear terms) have also been attempted but with only limited success.

We propose an alternative approach. First observe that the costate in the solution of the TPBVP (without the transversality condition) may be arbitrarily scaled:

Proposition 1 If $(x(t), \lambda(t))$ is a solution of (15), then so is $(x(t), \alpha \lambda(t))$ for any positive constant $\alpha$.
Proof: Let $(x(t), \lambda(t))$ be a solution of (15). For any $\alpha>0$,

$$
\frac{u_{\max }}{2}\left(1-\operatorname{sgn}\left[\lambda^{T} f_{1}(x)\right]\right)=\frac{u_{\max }}{2}\left(1-\operatorname{sgn}\left[\alpha \lambda^{T} f_{1}(x)\right]\right) .
$$

Hence $x$ also satisfies

$$
\dot{x}=f_{0}(x)+f_{1}(x) \frac{u_{\max }}{2}\left(1-\operatorname{sgn}\left[\alpha \lambda^{T} f_{1}(x)\right]\right), x(0)=x_{0} .
$$

Now scale both sides of the co-state equation in (15) by $\alpha$, we get

$$
\alpha \dot{\lambda}=f_{2}\left(x, \frac{u_{\max }}{2}\left(1-\operatorname{sgn}\left[\alpha \lambda^{T} f_{1}(x)\right]\right)\right) \alpha \lambda
$$

which is the same as the co-state equation in (15) with $\lambda$ replaced by $\alpha \lambda$. Hence (15) is satisfied by $(x(t), \alpha \lambda(t))$.

This Proposition states that if the initial co-state $\lambda(0)$ is scaled by a positive constant $\alpha$, the co-state trajectory $\lambda(t)$ will also be scaled by $\alpha$, but the state trajectory $x(t)$ will not change. Suppose $\left(\lambda(0), t_{f}\right)$ is found to satisfy the terminal state condition. If the transversality condition (14) is satisfied, then we have the optimal solution. Otherwise, $\lambda(0)$ may be scaled by $\alpha$,

$$
\alpha=-\frac{1}{\lambda\left(t_{f}\right)^{T}\left(\dot{x}\left(t_{f}\right)-\dot{x}_{r}\left(t_{f}\right)\right)}
$$

to satisfy (14). Since $\|\lambda(0)\|$ may be found using the result above, we may arbitrarily choose $\|\lambda(0)\|=1$ for the solution of TPBVP.

This leads to the following algorithm to solve the minimum time control problem:

For $\phi \in[0,2 \pi)$, set $\lambda(0)=[\cos (\phi) \sin (\phi)]^{T}$. Find the convergence time $T_{c}(\phi)$ from

$$
T_{c}(\phi)=\min \left\{\arg \min _{t}\left\|x(t)-x_{r}(t)\right\|<\varepsilon, T_{\max }\right\}
$$

where $\varepsilon$ is the convergence criterion and $T_{\max }$ is the upperbound for $t_{f}$ (which could be chosen as the baseline entrainment time). The minimum entrainment time $t_{f}$ is then given by

$$
t_{f}=\min _{\phi} T_{c}(\phi)
$$

The function $T_{c}(\phi)$ is multi-modal. To compute its minimum, we first coarsely discretize the $2 \pi$ interval and sequentially compute $T_{c}(\phi)$ by forward simulation to bracket the minimum. A unimodal line search is then applied to hone in on the solution. To reduce computation time, $T_{\max }$ may be lowered to the smallest convergence time of prior $\phi$ values.

If $\Gamma \triangleq \lambda^{T} f_{1}(x) \equiv 0$ on a trajectory of positive length, the optimal control is singular and cannot be determined from 
(13). To evaluate the possible existence of a singular arc, we apply the procedure from [38]. By setting both $\Gamma$ and its first derivative to zero, we have

$$
\lambda^{T} \underbrace{\left[f_{1}(x)\left[f_{0}, f_{1}\right](x)\right]}_{\triangleq L(x)}=0
$$

where $\left[f_{0}, f_{1}\right]$ denotes the Lie bracket operation. For a nontrivial solution of $\lambda$ to exist, $L(x)$ must be singular. The solution of $\varphi(x) \triangleq \operatorname{det}(L(x))=0$ is a curve in the state space. To keep the state trajectory on this curve, $u$ must be chosen such that $\dot{\varphi}(x(t))=0$. Substitute in the state equation (2), we solve the required $u$ :

$$
u^{(s)}=-\left(\nabla_{x} \varphi(x) f_{0}(x) / \nabla_{x} \varphi(x) f_{1}(x)\right)
$$

where $\nabla_{x} \varphi=\left[\begin{array}{ll}\frac{\partial \varphi}{\partial x_{1}} & \frac{\partial \varphi}{\partial x_{2}}\end{array}\right]$. The segment of the curve that can be traversed using a feasible input corresponds to $u^{(s)} \in$ $\left[0, u_{\max }\right]$. To numerically determine the optimality of these candidate singular arc segments, we apply the following procedure:

1 . For a given starting point $x^{(s)}$ on the segment, find $\lambda$ in the null space of $L^{T}\left(x^{(s)}\right)$. As discussed before, the magnitude of $\lambda$ does not affect the optimal trajectory. However, there are two choices, pointing in the opposite directions. To find the right $\lambda$, we use Kelley's necessary condition for singular arc optimality $[35,39]$,

$$
\frac{\partial}{\partial u}\left(\frac{d^{2}}{d t^{2}}\left(\frac{\partial H}{\partial u}\right)\right)=\lambda^{T}\left[\left[f_{1}, f_{0}\right], f_{1}\right](x) \leq 0 .
$$

Denote the co-state $\lambda$ that satisfies the above condition as $\lambda^{(s)}\left(x^{(s)}\right)$.

2. Integrate $x^{(s)}$ and $\lambda^{(s)}\left(x^{(s)}\right)$ backwards in time using (2), (12), and (13) with either $u=0$ or $u=u_{\max }$. One of them will depart from the segment and the other immediately returning to the segment. Continue integrating the departing trajectory backward until the solution intersects with the open loop entrained orbit. This is the initial condition that would reach the segment at the point chosen at step 1.

3 . From the entry point on the segment in step 1 , integrate $x$ and $\lambda$ forward using (2) and (12) with $u=u^{(s)}$ from (21), until it is no longer feasible to obey the input bounds.

4. From the point of exit, switch to the non-singular control (13) and integrate forward until the solution intersects the open loop entrained orbit again.

5. Let the point of intersection be the terminal point (i.e., the actual and reference trajectories coincide) and integrate backwards using the reference equation (3) for the combined times from steps 2, 3, and 4, to locate the starting condition of the reference trajectory.

6 . From the initial state in step 2 and the initial reference state in step 5, we can compute the optimal bang-off control solution. Comparing the convergence times using the bang-off control versus the singular control allows us to determine if a singular optimal control exist.

It is difficult to make a general statement about the existence of singular optimal control, but we may numerically evaluate the possibility for specific scenarios. As an example, consider the case of $50 \%$ duty cycle open loop entrainment reference trajectory and $u_{\max }=0.2392$. For this case, there are two small candidate singular arc segments, one inside the open loop periodic orbit and one outside, shown in blue in Figure 3. We then determine $\lambda^{(s)}$ that satisfies the Kelley's condition (22). For a number of points on these segments, we integrate backwards as in step 2 above $\left(u=u_{\max }\right.$ causes departure from the segments). Only the segment within the open loop periodic orbit intersects with the orbit. Using the procedure described above, we found the singular control generally produces the same convergence time as (or slightly worse than) our bang-off optimal control. An example case is shown in Figure 3; the backward trajectory is in green dash and forward trajectory is in black, connected by the candidate singular arc. Figure 4 shows the time plots of the state trajectories (for $x_{1}$ and $x_{r_{1}}$ only) and the light input trajectories for the singular input and the bang-off input cases. The inputs differ on the singular arc, but the state trajectories remain very close. Though we cannot state this in general, based on our numerical simulation for these cases, we have not encountered the situation where a singular control outperforms the bang-off control.

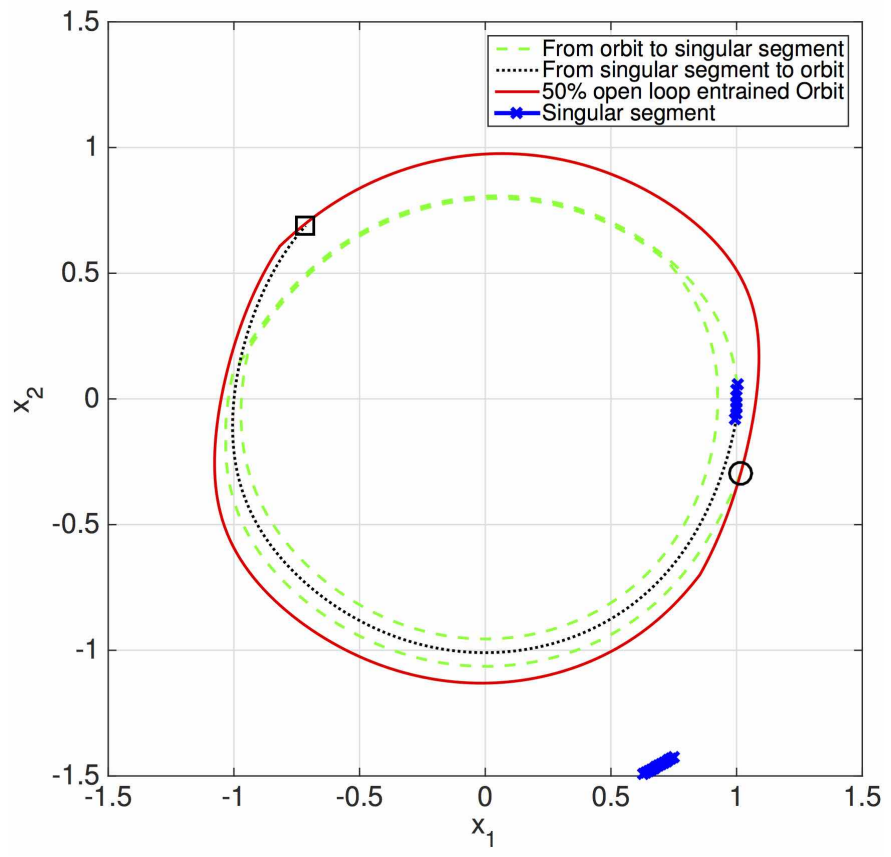

Fig. 3. A singular optimal control obtained by integrating backward and forward from a point on the candidate singular arc. The intersection with the open loop entrainment (50\% duty cycle in this example) periodic solution gives the singular trajectory (from the circle to the square). 

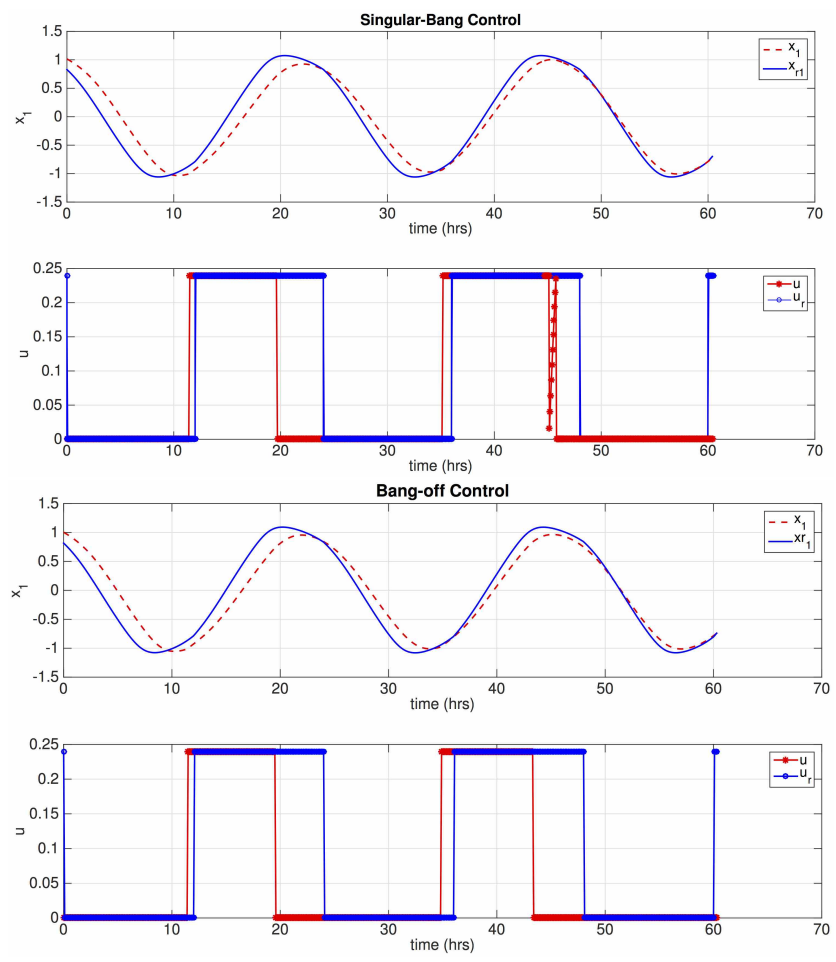

Fig. 4. Comparison between a singular trajectory and a bang-off optimal trajectory starting with the same initial condition. The convergence performance is nearly identical.

\subsection{Feedback Entrainment}

Compared with the open loop optimal control, feedback control has the potential to reject environmental disturbances and tolerate some level of modeling error. We pose the feedback entrainment problem as a reference trajectory tracking problem with light intensity as the input and the full circadian state $x$ for feedback (using the normalized core body temperature measurement) to drive $x(t) \rightarrow x_{r}(t)$ as $t \rightarrow \infty$. The human body temperature and other biometric sensors provide the possibility of real time estimation of the circadian state variables [31].

From Section 3.1, we know with $u=u_{r}, x$ converges to the reference trajectory $x_{r}$ asymptotically. Therefore, we choose the light input as

$$
u=u_{r}+\delta u
$$

with $\delta u \in\left[-u_{r}, u_{\max }-u_{r}\right]$ to add a feedback term to speed up convergence. This additional term can only add light, up to $u_{\max }$, when the ambient light is absent, and can block the ambient light when it is on.

First consider $u=u_{r}$. From the harmonic analysis, we know the periodic orbit is stable, therefore $\Phi(T)^{T} \Phi(T)-I$ is negative definite. Hence, the quadratic Lyapunov function

$$
V=\left\|x-x_{r}\right\|^{2}
$$

is strictly decreasing in each period:

$$
\begin{aligned}
& V(t+T)-V(t) \\
& =\left(x(t)-x_{r}(t)\right)^{T}\left(\Phi(T)^{T} \Phi(T)-I\right)\left(x(t)-x_{r}(t)\right) \\
& <\left(x(t)-x_{r}(t)\right)^{T} Q\left(x(t)-x_{r}(t)\right)
\end{aligned}
$$

where $Q$ is negative definite. Adding in the $\delta u$ term and use the same Lyapunov function, we have

$$
\begin{aligned}
V(t+T)-V(t) & =\left(x(t)-x_{r}(t)\right)^{T} Q\left(x(t)-x_{r}(t)\right) \\
& +\int_{t}^{t+T}\left(x(s)-x_{r}(s)\right)^{T} f_{1}(x(s)) \delta u(s) d s
\end{aligned}
$$

Choose

$$
\delta u= \begin{cases}u_{\max }-u_{r} & \text { if } f_{1}^{T}(x)\left(x-x_{r}\right) \leq 0 \\ -u_{r} & \text { if } f_{1}^{T}(x)\left(x-x_{r}\right)>0\end{cases}
$$

then $\dot{V}(t)$ remains negative definite in each period and the asymptotic convergence is not affected. However, the additional opportunistic light injection (when $f_{1}^{T}(x)\left(x-x_{r}\right) \leq 0$ ) or reference light blockage (when $f_{1}^{T}(x)\left(x-x_{r}\right)>0$ ) speeds up convergence (i.e., making $\dot{V}$ more negative). Note that feedback control law means that the input vector field, $f_{1}$ is in the opposite direction of the error vector $x-x_{r}$, i.e., if current circadian phase is ahead of the reference phase and the input vector field delays the phase, then use the maximum light input. Similarly, if the current circadian error is in the same direction of the input vector field, then use the minimum amount of light. The phase response curve has been used to implement lighting control in this manner [18]. If the phase needs to catch up and the light input causes phase advance (according to the PRC), then maximum light input should be used, otherwise, use the minimum light input. Conversely, if the phase needs to be delayed and light input at that instant causes delay, then maximum light is used, otherwise use the minimum light input.

The above stability argument is local in nature, based on the approximate harmonic analysis. The true closed loop stability may be verified using the reachable set analysis from hybrid system analysis [40]. The numerical result of this approach has been reported in [30].

We can modify the feedback algorithm to allow only blockage of the destination ambient light, i.e.,

$$
\delta u=\left\{\begin{array}{ll}
0 & \text { if } f_{1}^{T}(x)\left(x-x_{r}\right) \leq 0 \\
-u_{r} & \text { if } f_{1}^{T}(x)\left(x-x_{r}\right)>0
\end{array} .\right.
$$

The stability argument remains the same, and we expect slower convergence. However, the advantage is that this scheme removes the requirement of controllable artificial lighting, replacing with just circadian-light blocking sunglasses at select times. We call this the subtractive feedback entrainment. The circadian light blockage may be achieved 
by selectively removing the short wavelength component of daylight using optical filters, such as goggles and shades, while the long wavelength component light can still be used to enable vision.

\subsection{Periodic Entrainment}

As described in Section 3.1, the harmonic balance analysis is used to approximate the periodic solution when the input is periodic. When the limit cycle is stable, the approximate periodic orbit is close to the exact solution obtained via simulation as illustrated in Figures 5a-5c. For the unstable limit cycle case, the harmonic balance equation has large residue error and the approximation is no longer close as shown in Figure $5 \mathrm{~d}$. We can predict the stability of the periodic solution based on the linearized dynamics about the approximate solution. The result is shown Figure $6 a$, including the eigenvalues. The prediction is mostly verified by simulation except for two boundary cases, $(T, d)=(23,10 \%)$ and $(24,70 \%)$ ( $d$ is the duty cycle) where the eigenvalues of $\Phi(T)$ are stable but eigenvalues of $\Phi(T)^{T} \Phi(T)$ are not. Note that for $T=24$, beyond $60 \%$ duty cycle $(\approx 15$ hours of light input in a day, which is not usual for waking hours in modern life), the periodic entrainment is no longer stable. The change in the oscillation is dramatic, with the circadian state almost quenched to the origin in the $70 \%$ case. Starting from the same initial condition $x=[1,0]^{T}$, the trajectories under $50 \%$ versus $70 \%$ periodic entrainment is dramatic, as shown in Figure 7. This certainly needs to be verified in human experiments, as it has potentially significant implications (e.g., reducing blue spectrum of lighting at night).

The duty cycle also affects the entrainment convergence rate. With convergence threshold set at $\left\|x-x_{r}\right\|<0.1$, the entrainment times for $T=24 \mathrm{hr}$ and different duty cycle and phase shifts are shown in Figure 8. Not surprisingly, the higher duty cycle results in faster convergence time, until the periodic solution becomes unstable (at $70 \%$ and beyond). Note that the dominant (slower) eigenvalue of the linearized return map in Figure 6a also becomes faster with increasing duty cycle.

As harmonic balance method is an approximation method, it may generate wrong predictions in certain cases, e.g., at the boundary of stability as in Figure 6a, and also for low intensity light input. It is known that low intensity periodic light input may lead to phase misalignment [41]. This mean that a periodic orbit with the driving period does not exist. However, the low input magnitude means small fluctuation of the solution about some average orbit, which may inadvertently be classified as a stable periodic orbit by the harmonic balance method. As an example, consider the $30 \%$ input duty cycle. The comparison between the harmonic balance prediction versus the actual stability obtained vias simulation is shown in Figure 6b. Indeed, for 95lux and below (1\% of our imposed saturation level of 9500lux), harmonic balance makes the wrong prediction.
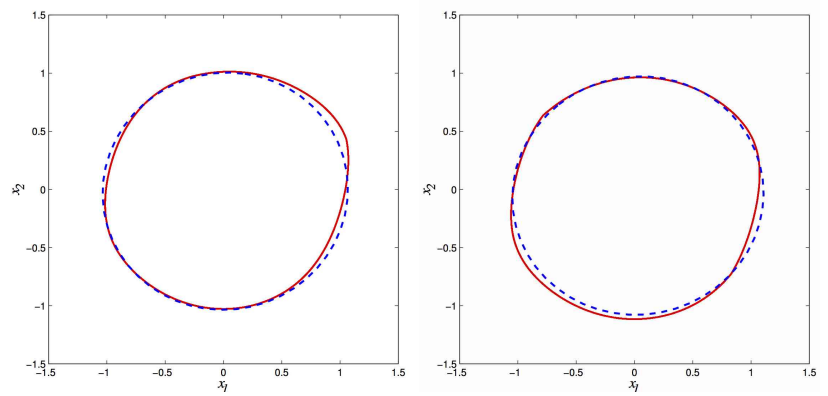

(a) $T=24 \mathrm{hr}, 20 \%$ duty cycle

(b) $T=24 \mathrm{hr}, 50 \%$ duty cycle

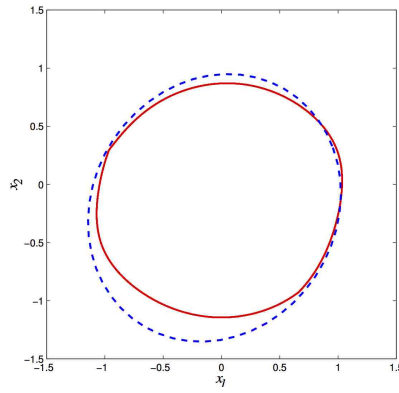

(c) $T=28 \mathrm{hr}, 50 \%$ duty cycle

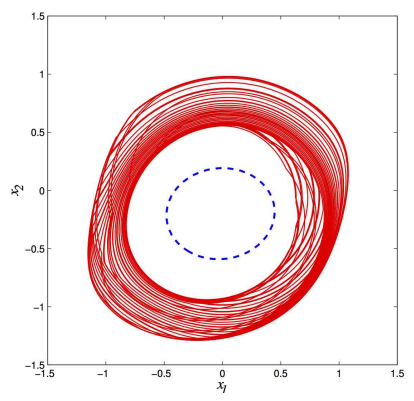

(d) $T=24 \mathrm{hr}, 80 \%$ duty cycle
Fig. 5. Comparison between approximate periodic orbit and exact periodic orbit for different duty cycles and periods.

\subsection{Optimal Entrainment}

As described in Section 3.2, to solve the TPBVP associated with the minimum time entrainment problem, we only need to search for the initial co-state on the unit circle, i.e., parameterize it as $\lambda(0)=[\cos (\phi) \sin (\phi)]^{T}$. For each $\phi \in[0,2 \pi)$, propagate the state and co-state equations forward until tracking is achieved, i.e. $x(t)=x_{r}(t)$ for some $t=t^{*}(\phi)$, or some upper limit is reached, $t=T_{\max }$. The minimum time is then given by $\min _{\phi} t^{*}(\phi)$. The optimal trajectories and the light input for the 12-hour time shift is illustrated in Figures 9. For the simulation comparison, both $x(0)$ and $x_{r}(0)$ are on the same periodic solution separated by the specified phase difference (between the corresponding periodic input light signal). The upper bound is set at $T_{\max }=240 \mathrm{hr}$ or 10 days since we know periodic entrainment (with $50 \%$ duty cycle) is no more than that in the worst case. For the 6-hour phase lag (going from east to west), The minimum entrainment time $t_{f}=\min _{\phi} T_{c}(\phi)$ is about 34 hours and the corresponding $\phi$ is $0.67 \mathrm{rad}$. The optimal light input, the corresponding state trajectories, and the local light pattern for different specified phase delays are shown in Figure 9.Additional light input beyond the destination local lighting are used to add phase delay (corresponding physically to the suppression of the onset of Melatonin), though this strategy does not take into account of the need for sleep. Some blockage of ambient lighting is also needed at times to allow for phase advance. Figure 10 summarizes the entrainment times for different target phase shifts and entrainment strategies under $50 \%$ duty cycle. 


\begin{tabular}{|c|c|c|c|c|c|c|c|c|}
\hline \multirow[t]{2}{*}{ Period } & \multicolumn{8}{|c|}{ Duty Cycle } \\
\hline & $10 \%$ & $20 \%$ & $30 \%$ & $40 \%$ & $50 \%$ & $60 \%$ & $70 \%$ & $80 \%$ \\
\hline 23 & $\begin{array}{c}{[.96, .27]} \\
{[1.18, .06]}\end{array}$ & & & & & & & \\
\hline 24 & $\begin{array}{c}{[.91, .04]} \\
{[.90,0]}\end{array}$ & $\begin{array}{c}{[.84,02]} \\
{[.81,0]}\end{array}$ & $\begin{array}{c}{[.78, .01]} \\
{[.75,0]}\end{array}$ & $\begin{array}{c}{[.74, .01]} \\
{[.70,0]}\end{array}$ & $\begin{array}{c}{[.70, .01]} \\
{[.68,0]}\end{array}$ & $\begin{array}{c}{[.70, .02]} \\
{[.63,0]}\end{array}$ & $\begin{array}{l}{[.95, .09]} \\
{[1.02,0]}\end{array}$ & \\
\hline 25 & $\begin{array}{c}{[.86, .04]} \\
{[.76,0]}\end{array}$ & $\begin{array}{c}{[.85, .02]} \\
{[.76,0]}\end{array}$ & $\begin{array}{c}{[.83, .01]} \\
{[.73,0]}\end{array}$ & $\begin{array}{l}{[.76,0]} \\
{[.67,0]}\end{array}$ & $\begin{array}{l}{[.68,0]} \\
{[.56,0]}\end{array}$ & $\begin{array}{l}{[.60,0]} \\
{[.48,0]}\end{array}$ & $\begin{array}{c}{[.56, .01]} \\
{[.42,0]}\end{array}$ & $\begin{array}{c}{[.95,05]} \\
{[1.0,0]}\end{array}$ \\
\hline 26 & & $\begin{array}{l}{[.73, .2]} \\
{[.59,0]}\end{array}$ & $\begin{array}{c}{[.68, .01]} \\
{[.48,0]}\end{array}$ & $\begin{array}{l}{[.66,0]} \\
{[.45,0]}\end{array}$ & $\begin{array}{l}{[.61,0]} \\
{[.41,0]}\end{array}$ & $\begin{array}{l}{[.53,0]} \\
{[.33,0]}\end{array}$ & $\begin{array}{l}{[.46,0]} \\
{[.27,0]}\end{array}$ & $\begin{array}{l}{[.48,0]} \\
{[.25,0]}\end{array}$ \\
\hline 27 & & & $\begin{array}{c}{[.69, .01]} \\
{[.57,0]}\end{array}$ & $\begin{array}{l}{[.59,0]} \\
{[.35,0]}\end{array}$ & $\begin{array}{l}{[.54,0]} \\
{[.31,0]}\end{array}$ & $\begin{array}{l}{[.48,0]} \\
{[.26,0]}\end{array}$ & $\begin{array}{l}{[.43,0]} \\
{[.22,0]}\end{array}$ & $\begin{array}{l}{[.44,0]} \\
{[.24,0]}\end{array}$ \\
\hline 28 & & & & $\begin{array}{c}{[.72, .01]} \\
{[.60,0]}\end{array}$ & $\begin{array}{l}{[.61,0]} \\
{[.37,0]}\end{array}$ & $\begin{array}{l}{[.56,0]} \\
{[.33,0]}\end{array}$ & $\begin{array}{l}{[.53,0]} \\
{[.31,0]}\end{array}$ & $\begin{array}{l}{[.57,0]} \\
{[.36,0]}\end{array}$ \\
\hline 29 & & & & & $\begin{array}{c}{[.96, .02]} \\
{[.93,0]}\end{array}$ & $\begin{array}{l}{[.82,0]} \\
{[.68,0]}\end{array}$ & $\begin{array}{l}{[.80,0]} \\
{[.65,0]}\end{array}$ & $\begin{array}{l}{[.87,0]} \\
{[.79,0]}\end{array}$ \\
\hline
\end{tabular}

(a) Stable periodic entrainment with respect to period $T$ and duty cycle with light intensity $I_{\max }=9500 \mathrm{Lux}$

\begin{tabular}{|c|c|c|c|c|c|c|c|}
\hline \multirow[t]{2}{*}{ Light Intensity } & \multicolumn{7}{|c|}{ Period } \\
\hline & 23 hrs & $24 \mathrm{hrs}$ & 25 hrs & 26 hrs & $27 \mathrm{hrs}$ & $28 \mathrm{hrs}$ & $29 \mathrm{hrs}$ \\
\hline 0.95 Lux & & $\begin{array}{c}{[0.820 .02]} \\
{[0.760]}\end{array}$ & $\begin{array}{c}{[0.840 .01]} \\
{[0.740]}\end{array}$ & $\begin{array}{c}{[0.850 .007]} \\
{[0.760]}\end{array}$ & $\begin{array}{c}{[0.880 .01]} \\
{[0.780]}\end{array}$ & & \\
\hline 9.5 Lux & & {$\left[\begin{array}{l}{[0.820 .02]} \\
{[0.760]}\end{array}\right.$} & {$\left[\begin{array}{c}{[0.840 .01]} \\
{[0.7400]}\end{array}\right.$} & {$\left[\begin{array}{c}{[0.850 .007]} \\
{[0.760]}\end{array}\right.$} & $\begin{array}{c}{[0.880 .01]} \\
{[0.7800]}\end{array}$ & & \\
\hline 95 Lux & & $\begin{array}{c}{[0.820 .02]} \\
{[0.750]}\end{array}$ & $\begin{array}{c}{[0.840 .01]} \\
{[0.740]}\end{array}$ & $\begin{array}{c}{[0.850 .007]} \\
{[0.7600]}\end{array}$ & 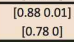 & & \\
\hline 950 Lux & & $\begin{array}{c}{[0.820 .02]} \\
{[0.750]}\end{array}$ & $\begin{array}{c}{[0.840 .01]} \\
{[0.730]}\end{array}$ & $\begin{array}{c}{[0.830 .007]} \\
{[0.730]}\end{array}$ & $\begin{array}{c}{[0.870 .01]} \\
{[0.7600]}\end{array}$ & & \\
\hline 9500 Lux & & $\begin{array}{c}{[0.780 .01]} \\
{[0.750]}\end{array}$ & $\begin{array}{c}{[0.830 .01]} \\
{[0.730]}\end{array}$ & $\begin{array}{c}{[0.680 .01]} \\
{[0.480]}\end{array}$ & $\begin{array}{c}{[0.690 .007]} \\
{[0.570]}\end{array}$ & & \\
\hline
\end{tabular}

(b) Stable periodic entrainment with respect to period $T$ and light intensity with duty cycle $=30 \%$ Lux

Fig. 6. Stable periodic entrainment for specified period and duty cycle. Top row shows eigenvalues of $\Phi(T)$, bottom row shows eigenvalues of $\Phi(T)^{T} \Phi(T)$. The green background indicates stability verified by simulation. The red background indicates stable $\Phi(T)$ but unstable in simulation.
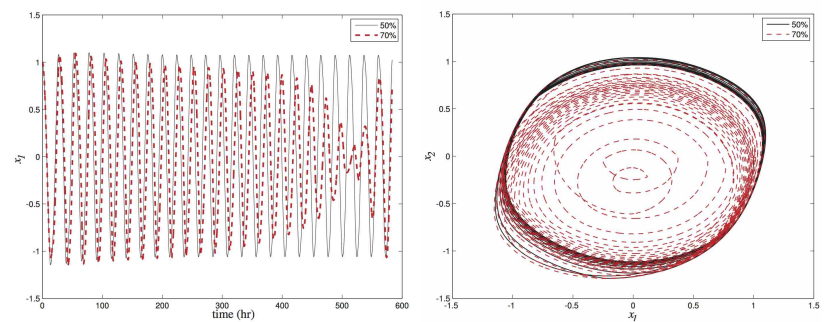

Fig. 7. Comparison of the circadian trajectories, $x_{1}$ vs. time (left) and in phase plane (right), under $50 \%$ and $70 \%$ periodic entrainment for $T=24 \mathrm{hr}$.

The optimal solution does vary somewhat with the start time, as indicated in Table 2. However, the basic strategy remains the same - the optimal strategy tries to emulate the destination lighting pattern but the light period for the first few days are extended to add delays.

The effect of light intensity is relatively small as shown in Table 1. The maximum intensity that we are using now, 9500lux, nearly saturates the $L$ process, so having strong intensity does not significantly change the solution. If we

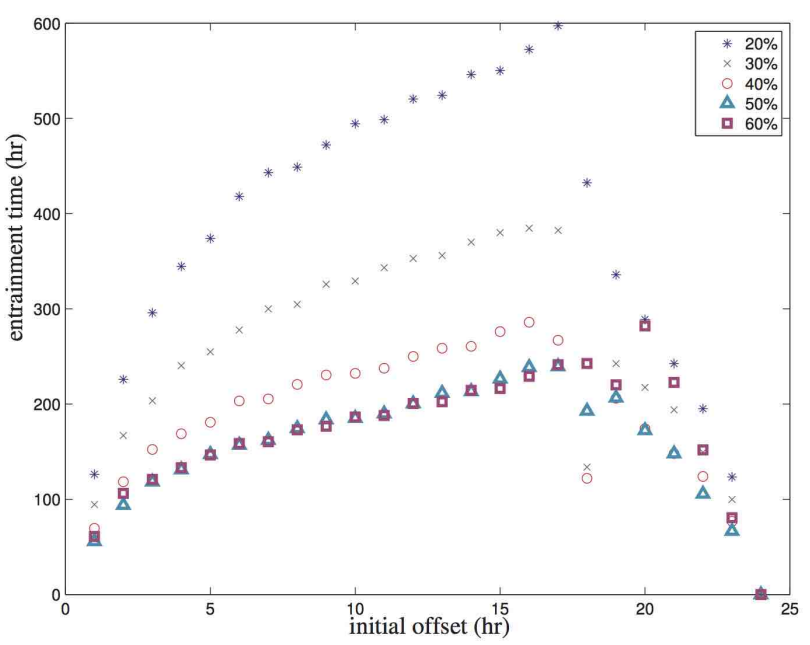

Fig. 8. Periodic entrainment time as a function of the phase shift and duty cycle for $T=24 \mathrm{hr}$

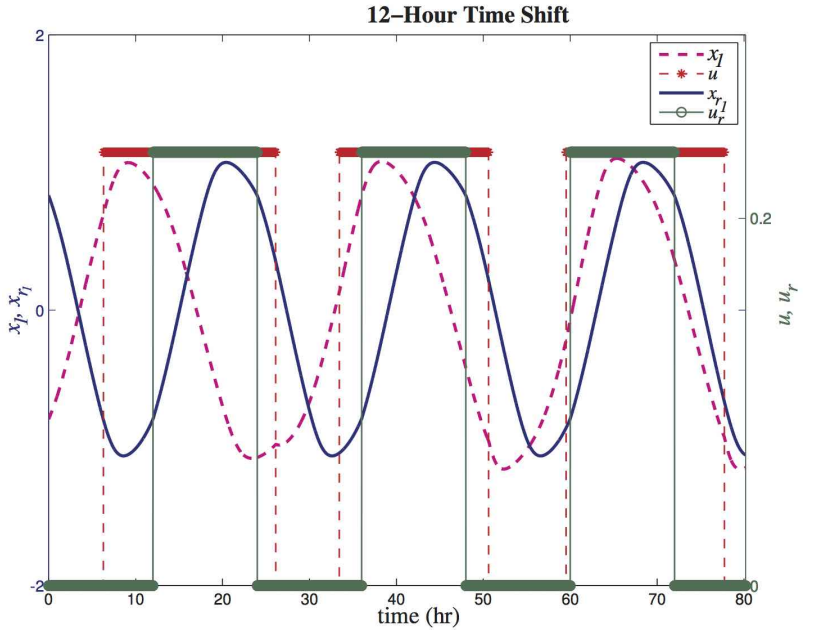

Fig. 9. Minimum time control and state trajectories for 12 hour time shift

reduce the maximum light intensity to 5000lux, the entrainment time is only increased slightly.

Table 1

\begin{tabular}{|l|c|c|c|}
\hline$I_{\max }$ & $6 \mathrm{hr}$ & $12 \mathrm{hr}$ & $18 \mathrm{hr}$ \\
\hline $5000 l u x$ & $34.9 \mathrm{hr}$ & $66.5 \mathrm{hr}$ & $98.7 \mathrm{hr}$ \\
\hline $95001 \mathrm{ux}$ & $34.0 \mathrm{hr}$ & $64.9 \mathrm{hr}$ & $97.1 \mathrm{hr}$ \\
\hline $10000 \mathrm{lux}$ & $33.9 \mathrm{hr}$ & $64.2 \mathrm{hr}$ & $97.1 \mathrm{hr}$ \\
\hline
\end{tabular}

Effect of maximum light intensity on optimal entrainment time for 6 hour, 12 hour, 18 hour phase delay

\subsection{Feedback Entrainment}

The feedback entrainment selectively inject or block ambient light $u_{r}$ to speed up convergence. As expected, both the active lighting feedback entrainment and subtractive entrainment improves the convergence rate over the periodic en- 


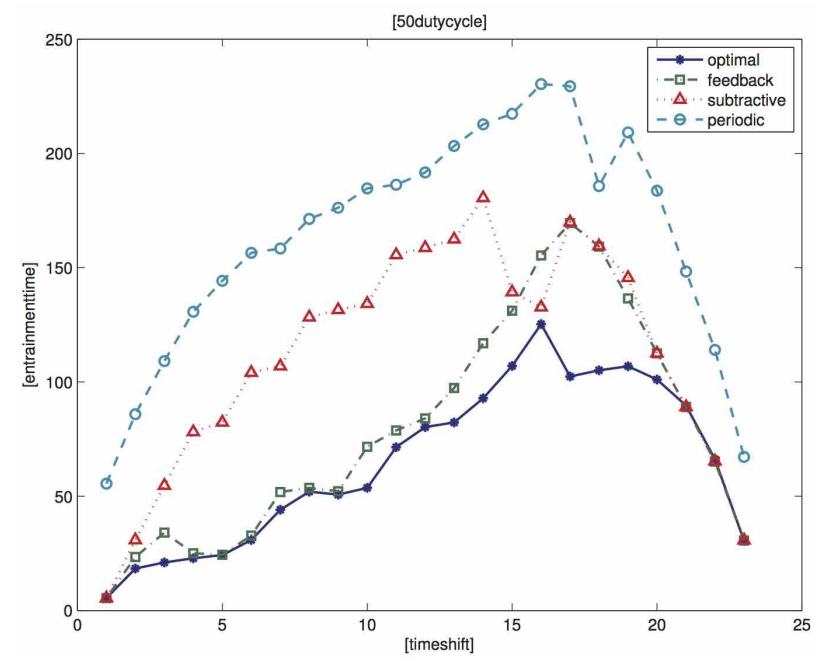

Fig. 10. Comparison of the time cost as a function of the specified phase shift under 12h/12h-light/dark periodic input (baseline), optimal entrainment, active feedback entrainment and subtractive feedback entrainment.

trainment. Figure 11 shows the tracking error convergence comparison for 6 hour delay, 12 hour delay, and 4 hour advance. The active feedback is most advantageous for the delay cases. For phase delay, subtractive feedback is not effective. In the phase advance case, subtractive feedback works equally well, and active light injection is hardly used. This is due to the fact that light input tends to delay, hence blockage of ambient light results in phase advance.

Figure 12 shows the state trajectory and light input for the periodic and feedback entrainment cases for the 12-hour time shift under $50 \%$ duty cycle. The feedback control reduces the circadian amplitude to speed up convergence. The trajectories for the subtractive case is shown in Figure 13 for the 6-hour phase advance (18-hour delay).

The entrainment times comparison for periodic, feedback, and subtractive entrainments for various duty cycles is summarized in Figure 14. The feedback case achieves same entrainment time for all duty cycles, demonstrate its ability to adjust for different conditions. The subtractive is most effective for higher duty cycle as there is more opportunity to block off ambient light. Note that for time shift of 18 hours (6 hour advance), the open loop periodic entrainment under $30 \%$ and $40 \%$ duty cycle performs slightly better than the feedback cases. This is due to the fact that the analysis is approximate, and some discrepancy from the prediction is

Table 2

\begin{tabular}{|l|c|c|c|}
\hline Start time & $6 \mathrm{hr}$ & $12 \mathrm{hr}$ & $18 \mathrm{hr}$ \\
\hline $3 \mathrm{pm}$ & $34.0 \mathrm{hr}$ & $64.9 \mathrm{hr}$ & $97.1 \mathrm{hr}$ \\
\hline $6 \mathrm{am}$ & $31.9 \mathrm{hr}$ & $72.1 \mathrm{hr}$ & $105.1 \mathrm{hr}$ \\
\hline $12 \mathrm{am}$ & $30.8 \mathrm{hr}$ & $61.3 \mathrm{hr}$ & $91.1 \mathrm{hr}$ \\
\hline
\end{tabular}

Effect of starting time (at the origin for the traveler) on optimal entrainment time for 6 hour, 12 hour, 18 hour phase delay expected.

Overall, the feedback and subtractive feedback control are stable and perform better than the periodic entrainment. These feedback algorithms are also easy to implement in the sense that if the model predicts that light input is helpful to reduce the phase difference to the target (may also use PRC to make this prediction), then apply the maximum active light input; conversely, if the light input increase the phase difference, then block of the light input.

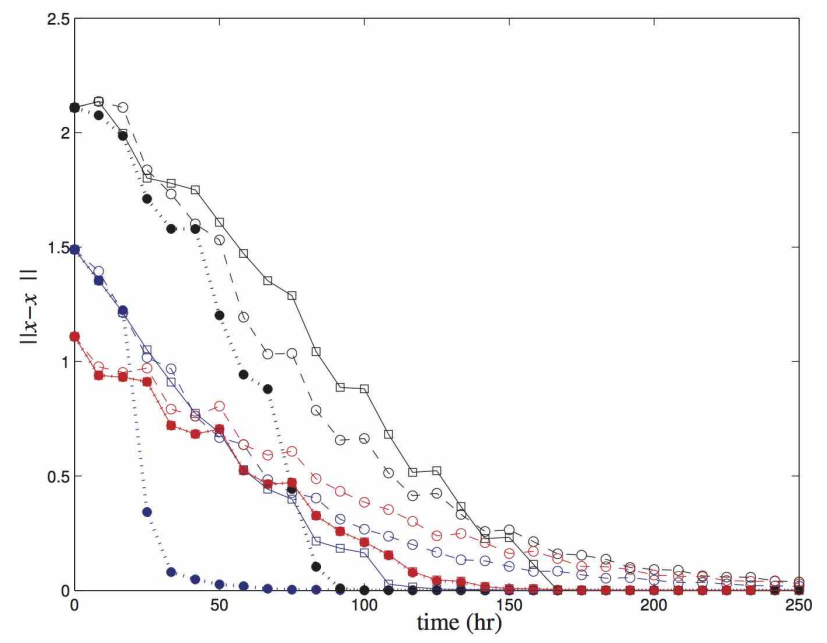

Fig. 11. Tracking error trajectories for periodic entrainment, feedback entrainment, and subtractive entrainment under $50 \%$ input duty cycle.

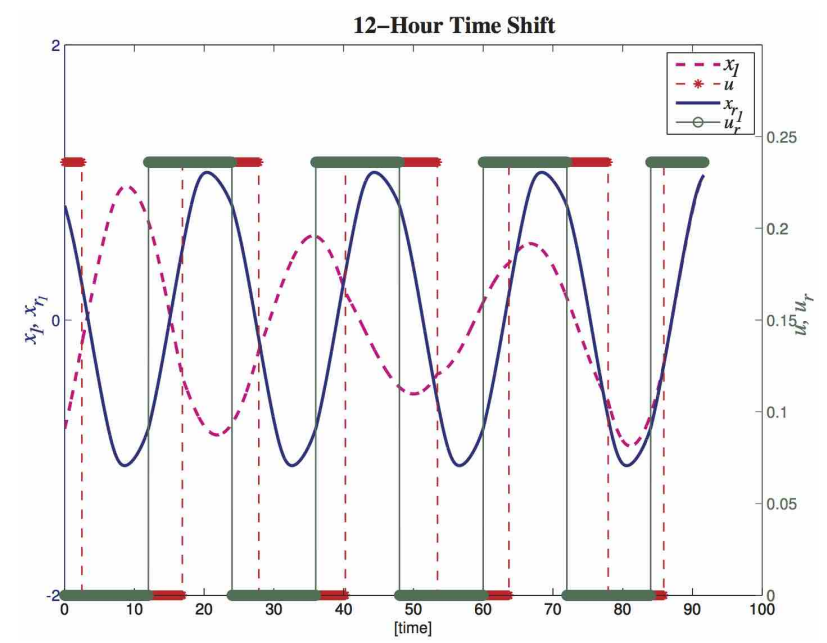

Fig. 12. Comparison between periodic entrainment and feedback entrainment: $x_{1}$ (left) and $u$ (right)

\subsection{Discussion}

Based on the entrainment time comparison shown in Figure 10, we make the following observations:

- Entrainment using active light input is most effective for phase delay. This is built into the input vector field, $f_{1}$ 


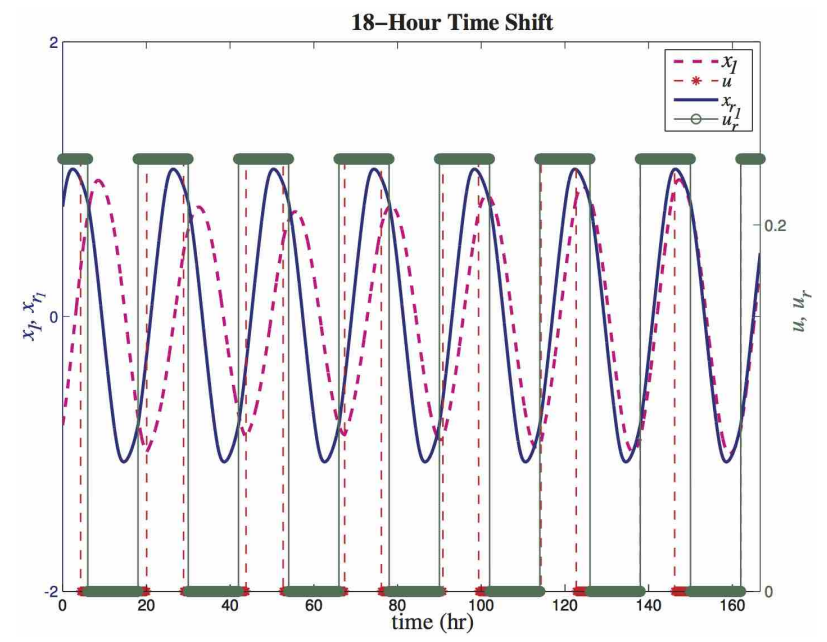

Fig. 13. Comparison between periodic entrainment and subtractive feedback entrainment: $x_{1}$ (left) and $u$ (right)

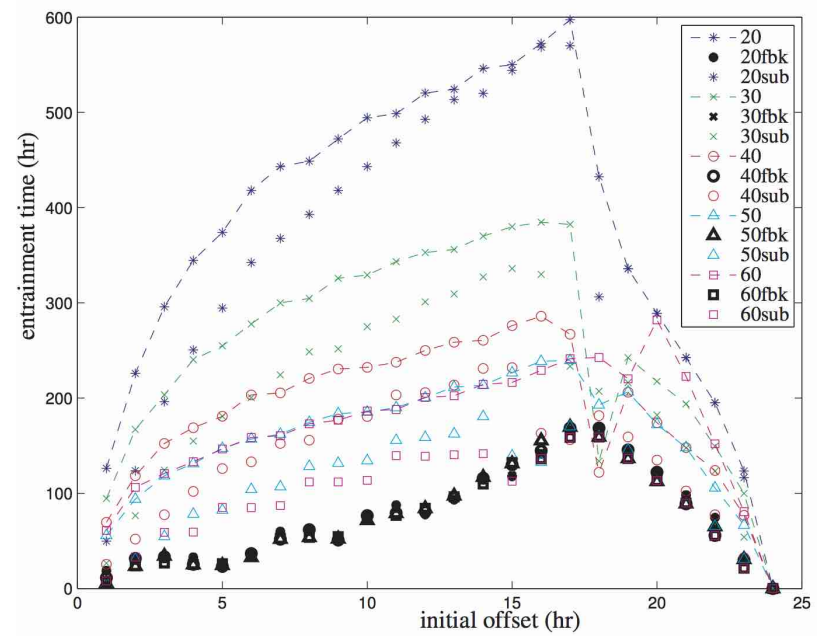

Fig. 14. Entrainment time comparison between periodic entrainment, feedback entrainment, and subtractive feedback entrainment for $20 \%-60 \%$ duty cycle

in (2), as shown in Figure 2 superimposed onto the limit cycle. Since the light input is non-negative, the effect of light is mostly to add delay to the oscillation, except for about the upper quarter of the limit cycle. This is not entirely surprising, since light (in the blue spectrum) tends to suppress the secretion of melatonin.

- Active light input is less effective in phase advance. This is the corollary of the observation above, due to the direction of the input vector field $f_{1}$. In this case, the subtractive strategy is more effective. By blocking the ambient light at appropriate times, the inherent delay due to the ambient light is reduced, leading to phase advance. For small phase advance (up to 4 hours), the optimal entrainment time is almost identical to the subtractive feedback case. The control strategies for both cases, optimal and subtractive, are almost identical. Both strategies consist of blocking the light input several hours before the onset of darkness at destination.

- Active entrainment based on minimum time control or feedback control are both effective in achieving phase delay entrainment, significantly improving over the baseline. For example, for the 12-hour phase shift, the entrainment time is reduced from about 8 days using the destination daylight to $2 \frac{1}{2}$ days using the optimal control. For the worst case 16-hour phase delay (or 8-hour phase advance), the improvement is the largest: 10 days vs. 3 days. For small phase advance, the optimal control just requires strategic blocking of the natural light.

- Subtractive feedback entrainment is optimal for small phase advances (up to 4-6 hours) but becomes ineffective for phase delays. This is due to the fact that light blockage only has a small window of opportunity in the circadian cycle to add delay: when there is daylight and the input vector field advances the phase (around late morning in traveler's clock).

- The dynamics in the phototransduction stage (the $L$ process) is much faster than that of the $P$-precess and the saturation in the $L$-process is already taken into account. Therefore, the effect of the phototransduction stage is minimal. When our optimal control based on $P$-process alone is applied to the full model that includes the $L$ process, the resulting trajectory is nearly identical. In the feedback case, because of the higher initial light drive, the convergence is actually faster, particularly for the more challenging phase advance cases.

\section{Conclusion}

This paper analyzes light-based circadian entrainment using the empirical nonlinear oscillator model of the human circadian system proposed by Kronauer. The entrainment process is formulated as a reference tracking problem, and the entrainment time, or the convergence time to the reference trajectory, is used as performance metric. Three types of entrainment strategies are considered: periodic entrainment, optimal entrainment, and feedback entrainment. The harmonic balance method is used to estimate the periodic orbit under periodic entrainment and to obtain the stability condition. The minimum time control establishes the theoretical lower bound on the entrainment time. We have developed a novel and efficient numerical solution strategy for the associated two-point boundary value problem. Feedback entrainment modifies the periodic entrainment by adding light when it is absent and blocking light when it is present. The Lyapunov function from the periodic entrainment is used to establish stability of the feedback strategies. Two types of feedback are considered, active light injection and blockage, and active light blocking only.

The simulation result shows the known effect that phase shift is not symmetrical in the phase lag versus phase advance directions. Active lighting control is effective in introducing phase lag (for going from east to west). Indeed, for the 12-hour phase shift, the minimum time solution is reduced from the baseline (using local light/dark cycle) 8.5 hours to 
2.5 hours. The basic strategy is to use light control beyond the local daylight period to introduce phase lag. For phase advance, active light control is of little value since its effect is mostly in phase lag. The entrainment time in active feedback case is larger than the optimal control case (especially for phase lags), but still improves significantly from the baseline. In the subtractive feedback case, the performance is near optimal for phase advances. Feedback strategy is attractive as it is robust under disturbance and modeling error than the open loop method. However, the full circadian state is needed for its implementation. We are currently investigating circadian state estimation by using output measurements.

\section{Acknowledgment}

This work was supported primarily by the National Science Foundation (NSF) Smart Lighting Engineering Research Center (EEC-0812056) and in part by the Center for Automation Technologies and Systems (CATS) under a block grant from the New York State Empire State Development Division of Science, Technology and Innovation (NYSTAR) contract C090145. The authors would also like to thank Drs. Mark Rea, Mariana Figueiro, and Andrew Bierman at the RPI Light Research Center for introducing this topic to the authors.

\section{Appendix}

\section{A Harmonic Analysis under Periodic Light Input}

When $u=0$, we use the first harmonic approximation of $x_{1}(t)=a \sin \omega t$. The first harmonic nonlinear approximation of $g(a \sin \omega t)$ is $\hat{g}=-\frac{\pi a^{3}\left(4 a^{4}-3\right)}{36}$. The harmonic balance is the complex equation, $a=\mu B^{T}(j \omega I-A)^{-1} B \hat{g}$ which solves for $a=1, \omega=2 \pi / 24.13=0.26$. The ration $\hat{g} / a$ is called the describing function of $g$.

When $u$ is a given periodic function of period $T, \omega=2 \pi / T$ is specified. In this case, we need to use the full state equation (2) with the following first order approximation:

$$
\begin{aligned}
& \hat{x}_{1}=a_{1} \sin \omega t+b_{1} \cos \omega t+c_{1} \\
& \hat{x}_{2}=a_{2} \sin \omega t+b_{2} \cos \omega t+c_{2} .
\end{aligned}
$$

Let $\hat{x}=\left[\begin{array}{ll}\hat{x}_{1} & \hat{x}_{2}\end{array}\right]^{T}$. The derivative $\dot{\hat{x}}$ is

$$
\begin{aligned}
& \dot{\hat{x}}_{1}=\omega\left(a_{1} \cos \omega t-b_{1} \cos \omega t\right) \\
& \dot{\hat{x}}_{2}=\omega\left(a_{2} \cos \omega t-b_{2} \cos \omega t\right) .
\end{aligned}
$$

To extract the harmonics of the input, we project the input onto the harmonic basis functions:

$$
\begin{aligned}
\hat{v} & =\hat{v}_{0}+\hat{v}_{s} \sin \omega t+\hat{v}_{c} \cos \omega t \\
\hat{v}_{0} & =\frac{1}{T} \int_{0}^{T} f_{1}(\hat{x}(t)) u(t) d t \\
\hat{v}_{s} & =\frac{2}{T} \int_{0}^{T} f_{1}(\hat{x}(t)) u(t) d t \sin \omega t \\
\hat{v}_{c} & =\frac{2}{T} \int_{0}^{T} f_{1}(\hat{x}(t)) u(t) d t \cos \omega t .
\end{aligned}
$$

The harmonic components of $g\left(x_{1}\right)$ may be similarly obtained:

$$
\begin{aligned}
\hat{g} & =\hat{g}_{0}+\hat{g}_{s} \sin \omega t+\hat{g}_{c} \cos \omega t \\
\hat{g}_{0} & =\frac{1}{T} \int_{0}^{T} g\left(\hat{x}_{1}(t)\right) d t \\
\hat{g}_{s} & =\frac{2}{T} \int_{0}^{T} g\left(\hat{x}_{1}(t)\right) d t \sin \omega t \\
\hat{g}_{c} & =\frac{2}{T} \int_{0}^{T} g\left(\hat{x}_{1}(t)\right) d t \cos \omega t .
\end{aligned}
$$

The harmonic balance equation follows the state equation (2):

$$
\dot{\hat{x}}=A \hat{x}+B \hat{g}+\hat{v} .
$$

The six component equations may be used to solve for the six unknowns $\left(a_{1}, b_{1}, c_{1}, a_{2}, b_{2}, c_{2}\right)$ for the approximate periodic solution $\hat{x}$. Note that since the system is time invariant, if for a time-shifted input $u\left(t+t_{a}\right)$, the state is also shifted $x\left(t+t_{a}\right)$ which means that the periodic orbit remains the same in the state space.

To determine the stability of the periodic solution, we linearize the state equation about the solution:

$$
\delta \dot{x}=\underbrace{\left(A+B g^{\prime}\left(B^{T} \hat{x}(t)\right) B^{T}+\frac{\partial f_{1}(\hat{x}(t))}{\partial x} u(t)\right)}_{\triangleq \hat{A}(t)} \delta x .
$$

Divide the period into $N$ intervals, the time-varying system may be approximated as a discrete time system:

$$
x_{d_{i}}=e^{\hat{A}((i-1) T / N)} x_{d_{i-1}}
$$

where $x_{d_{i}}=x(i T / N)$. The stability of the period- $T$ orbit is determined by the fundamental matrix $\Phi(T)$ which may be approximated by

$$
\Phi(T) \approx \prod_{i=1}^{N} e^{\hat{A}((i-1) T / N)}
$$

If all the eigenvalues of $\Phi(T)$ is within the unit circle, the periodic orbit is predicted to be stable. The eigenvectors of $\Phi(T)$ typically are in the tangential and normal direction of the orbit, with the smaller (faster) eigenvalue corresponding to the tangential direction and the larger (slower) eigenvalue for the normal direction. 


\section{References}

[1] Refinetti R. Circadian physiology. CRC Press, 2006.

[2] Russell G, Kreitzman L. Rhythms of Life: The biological clocks that Control the daily lives of every living thing. Yale University Press, 2005.

[3] Rea M, Bierman A, Figueiro M, Bullough J. A new approach to understanding the impact of circadian disruption on human health. Journal of Circadian Rhythm 2008; 6(7).

[4] Doyle, III F, Gunawan R, Bagheri N, Mirsky H, To T. Circadian rhythm: A natural, robust, multi-scale control system. Computers \& Chemical Engineering 2006; 30(10-12):1700-1711.

[5] Bechtel W, Abrahamsen A. Dynamic mechanistic explanation: computational modeling of circadian rhythms as an exemplar for cognitive science. Studies In History and Philosophy of Science Part A 2010; 41(3):321 - 333. Computation and Cognitive Science.

[6] Wever R. Virtual synchronization towards the limits of the range of entrainment. Journal of Theoretical Biology 1972; 36:119-132.

[7] Jewett M, Forger D, Kronauer R. Revised limit cycle oscillator model of human circadian pacemaker. Journal of Biological Rhythms 1999; 14(6):493-499.

[8] Kronauer R, Forger D, Jewett M. Errata: Quantifying human circadian pacemaker response to brief, extended, and repeated light stimuli over the phototopic range. Journal of Biological Rhythms 2000; 15(2):184-186.

[9] Murray JD. Mathematical Biology. Springer-Verlag: Berlin, 1993.

[10] Leloup J, Goldbeter A. A model for circadian rhythms in Drosophila incorporating the formation of a complex between the PER and TIM proteins. Journal of Biological Rhythms 1998; 13:70-87.

[11] Leloup J, Goldbeter A. Toward a detailed computational model for the mammalian circadian clock. Proceedings of National Academy of Science 2003; 100(12):7051-7056.

[12] Xie Z, Kulasiri D. Modelling of circadian rhythms in drosophila incorporating the interlocked PER/TIM and VRI/PDP1 feedback loops. Journal of Theoretical Biology 2007; 245:290-304.

[13] Klarsfeld A, Leloup J, Rouyer F. Circadian rhythms of locomotor activity in drosophila. Behavioural Processes 2003; 64:161-175.

[14] Forger D, Peskin C. A detailed predictive model of the mammalian circadian clock. Proceedings of National Academy of Science 2003; 100:14 806-14 811.

[15] Forger D, Kronauer R. Reconciling mathematical models of biological clocks by averaging on approximate manifolds. SIAM Journal on Applied Mathematics 2002; 62:1281-1296.

[16] Bamieh B, Giarré L. On discovering low order models in biochemical reaction kinetics. American Control Conference, New York, NY, 2007; $2702-2707$

[17] Dean, II D, Forger D, Klerman E. Taking the lag out of jet lag through model based schedule design. PLoS Computational Biology $2009 ; 5(6)$

[18] Bagheri N, Stelling J, Doyle, III F. Circadian phase entrainment via nonlinear model predictive control. International Journal of Robust and Nonlinear Control 2007; 17(17):1555-1571.

[19] Wang Y, Doyle, III F. On influences of global and local cues on the rate of synchronization of oscillator networks. Automatica 2011; 47(6): 1236 - 1242. Special Issue on Systems Biology.

[20] Efimov D. Phase resetting control based on direct phase response curve. Journal of Mathematical Biology 2011; 63(5):855-879.

[21] Zhang J, Wen JT, Julius A. Optimal and feedback control for light-based circadian entrainment. Proceedings of the 52nd IEEE Conference on Decision and Control, Florence, Italy, 2013; 26772682
[22] Jewett M, Rimmer D, Duffy J, Klerman E, Kronauer R, Czeisler C. Human circadian pacemaker is sensitive to light throughout subjective day without evidence of transients. American Journal of Physiology - Regulatory, Integrative and Comparative Physiology 1997; 273:1800-1809.

[23] Granada A, Herzel H. How to achieve fast entrainment? the timescale to synchronization. PLOS ONE 2009; 4(9):e7057.

[24] Mott C, Mollicone D, Van Wollen M, Huzmezan M. Modifying the human circadian pacemaker using model based predictive control. American Control Conference, Denver, CO, 2003; 453-458.

[25] Serkh K, Forger D. Optimal schedules of light exposure for rapidly correcting circadian misalignment. PLoS Computational Biology 2014; 10(4):e1003 523.

[26] Vidyasagar M. Nonlinear Systems Analysis. Prentice-Hall: NJ, 1978.

[27] Khalil H. Nolinear Systems. third edn., Prentice-Hall, 2002.

[28] Zhang J, Wen JT, Julius A, Bierman A, Figueiro M. Modeling of drosophila circadian system based on the locomotor activity. American Control Conference, San Francisco, CA, 2011.

[29] Hori Y, Takada M, Hara S. Biochemical oscillations in delayed negative cyclic feedback: Existence and profiles. Automatica 2013 49(9):2581 - 2590.

[30] Zhang J, Wen J, Julius A. Optimal circadian rhythm control with light input for rapid entrainment and improved vigilance. Proceedings of the 51st IEEE Conference on Decision and Control, Maui, HI, 2012; 3007-3012.

[31] Mott C, Dumont G, Boivin DB, Mollicone D. Model-based human circadian phase estimation using a particle filter. IEEE Transactions on Biomedical Engineering 2011; 58(5):1325-1336.

[32] Zhang J, Wen JT, Julius A. Adaptive circadian rhythm estimator and its application to locomotor activity. IEEE Signal Processing in Medicine and Biology Symposium, 2012; 1-6.

[33] Kronauer R, Forger D, Jewett M. Quantifying human circadian pacemaker response to brief, extended, and repeated light stimuli over the phototopic range. Journal of Biological Rhythms 1999; 14(6):501-516.

[34] Zhang J, A Bierman A, Wen JT, Julius A, Figueiro M. Circadian system modeling and phase control. Proceedings of the 49th IEEE Conference on Decision and Control, Atlanta, GA, 2010; 6058-6063.

[35] Bryson A, Ho Y. Applied Optimal Control. Hemisphere, 1975.

[36] Liang J, Chen Y, Meng Q, Fullmer R. Solving tough optimal control problems by network enabled optimization server (NEOS). Technical Report, School of Engineering, Utah State University and Chinese University of Hong Kong 2003.

[37] Xu X, Antsakalis PJ. Optimal control of switched systems based on parameterization of the switching instants. IEEE Transactions on Automatic Control 2004; 49(1):216.

[38] Liberzon D. Calculus of variations and optimal control theory: a concise introduction. Princeton University Press, 2011.

[39] Kelley HJ. A second variation test for singular extremals. AIAA Journal 1964; 2(8):1380-1382.

[40] Donze A, Krogh B, Rajhans A. Parameter synthesis for hybrid systems with an application to simulink models. Hybrid Systems: Computation and Control 2009; 5469:165-179.

[41] CIBA Foundation Symposium. Circadian Clocks and Their Adjustment. Wiley, 2008. 